\title{
Intraoperative MRI for newly diagnosed supratentorial glioblastoma: a multicenter-registry comparative study to conventional surgery
}

\author{
Amar S. Shah, MD, MPHS, ${ }^{1}$ Peter T. Sylvester, MD, MHA, MSCI, ${ }^{1}$ Alexander T. Yahanda, MS, ${ }^{1}$ \\ Ananth K. Vellimana, MD, ${ }^{1}$ Gavin P. Dunn, MD, PhD, ${ }^{1}$ John Evans, RN, ${ }^{1}$ Keith M. Rich, MD, ${ }^{1}$ \\ Joshua L. Dowling, MD, ${ }^{1}$ Eric C. Leuthardt, MD, ${ }^{1}$ Ralph G. Dacey, MD, ${ }^{1}$ Albert H. Kim, MD, PhD, ${ }^{1}$ \\ Robert L. Grubb, MD, ${ }^{1}$ Gregory J. Zipfel, MD, ${ }^{1}$ Mark Oswood, MD, PhD, ${ }^{2,3}$ \\ Randy L. Jensen, MD, PhD, ${ }^{4}$ Garnette R. Sutherland, MD, ${ }^{5}$ Daniel P. Cahill, MD, PhD, ${ }^{6}$ \\ Steven R. Abram, MD, ${ }^{7}$ John Honeycutt, MD, ${ }^{8}$ Mitesh Shah, MD, ${ }^{9}$ Yu Tao, MD, ${ }^{1}$ and \\ Michael R. Chicoine, MD'
}

1Department of Neurosurgery, Washington University School of Medicine, St. Louis, Missouri; '2Department of Radiology, University of Minnesota, Minneapolis, Minnesota; ${ }^{3}$ Allina Health, Minneapolis, Minnesota; ${ }^{4}$ Department of Neurosurgery, Huntsman Cancer Institute, University of Utah, Salt Lake City, Utah; ${ }^{5}$ Department of Clinical Sciences and Hotchkiss Brain Institute, University of Calgary, Alberta, Canada; ${ }^{\circ}$ Department of Neurosurgery, Massachusetts General Hospital, Boston, Massachusetts; ${ }^{7}$ Department of Neurosurgery, St. Thomas Hospital, Nashville, Tennessee; ${ }^{8}$ Department of Neurosurgery, Cook Children's Hospital, Fort Worth, Texas; and 'Department of Neurological Surgery, Goodman Campbell and Indiana University, Indianapolis, Indiana

OBJECTIVE Intraoperative MRI (iMRI) is used in the surgical treatment of glioblastoma, with uncertain effects on outcomes. The authors evaluated the impact of iMRI on extent of resection (EOR) and overall survival (OS) while controlling for other known and suspected predictors.

METHODS A multicenter retrospective cohort of 640 adult patients with newly diagnosed supratentorial glioblastoma who underwent resection was evaluated. iMRI was performed in 332/640 cases (51.9\%). Reviews of MRI features and tumor volumetric analysis were performed on a subsample of cases $(n=286 ; 110$ non-iMRI, 176 iMRI) from a single institution.

RESULTS The median age was 60.0 years (mean 58.5 years, range 20.5-86.3 years). The median OS was 17.0 months (95\% Cl 15.6-18.4 months). Gross-total resection (GTR) was achieved in 403/640 cases (63.0\%). Kaplan-Meier analysis of 286 cases with volumetric analysis for EOR (grouped into 100\%, 95\%-99\%, 80\%-94\%, and 50\%-79\%) showed longer OS for $100 \%$ EOR compared to all other groups $(p<0.01)$. Additional resection after iMRI was performed in $104 / 122$ cases (85.2\%) with initial subtotal resection (STR), leading to a $6.3 \%$ mean increase in EOR and a $2.2-\mathrm{cm}^{3}$ mean decrease in tumor volume. For iMRI cases with volumetric analysis, the GTR rate increased from 54/176 (30.7\%) on iMRI to 126/176 (71.5\%) postoperatively. The EOR was significantly higher in the iMRI group for intended GTR and STR groups ( $p=0.02$ and $p<0.01$, respectively). Predictors of GTR on multivariate logistic regression included iMRI use and intended GTR. Predictors of shorter OS on multivariate Cox regression included older age, STR, isocitrate dehydrogenase 1 (IDH1) wild type, no $0^{6}$-methylguanine DNA methyltransferase (MGMT) methylation, and no Stupp therapy. iMRI was a significant predictor of OS on univariate (HR 0.82, 95\% $\mathrm{Cl} 0.69-0.98 ; p=0.03)$ but not multivariate analyses. Use of iMRI was not associated with an increased rate of new permanent neurological deficits.

CONCLUSIONS GTR increased OS for patients with newly diagnosed glioblastoma after adjusting for other prognostic

ABBREVIATIONS ASA = American Society of Anesthesiologists; EOR = extent of resection; GTR = gross-total resection; IDH = isocitrate dehydrogenase; I-MiND = IMRIS Multicenter iMRI Neurosurgery Database; iMRI = intraoperative MRI; iUS = intraoperative ultrasound; KPS = Karnofsky Performance Scale; MGMT = $0^{6}-$ methylguanine DNA methyltransferase; $n P N D$ = new permanent neurological deficits; OS = overall survival; PFS = progression-free survival; $Q A L Y=$ quality-adjusted life year; STR = subtotal resection; 5-ALA = 5-aminolevulinic acid.

SUBMITTED February 1, 2019. ACCEPTED June 4, 2020.

INCLUDE WHEN CITING Published online October 9, 2020; DOI: 10.3171/2020.6.JNS19287. 
factors. IMRI increased EOR and GTR rate and was a significant predictor of GTR on multivariate analysis; however, iMRI was not an independent predictor of OS. Additional supporting evidence is needed to determine the clinical benefit of MRI in the management of glioblastoma.

https://thejns.org/doi/abs/10.3171/2020.6.JNS19287

KEYWORDS glioblastoma; intraoperative magnetic resonance imaging; treatment outcomes; registry; clinical research; neurosurgery; oncology

$\mathrm{G}$ LIOBLASTOMA (WHO grade IV glioma), the most common adult primary brain malignancy, has a median survival of 15 months and 5-year overall survival (OS) less than 5\%., Despite advances, glioblastoma treatments have failed to yield paradigm-shifting benefits as seen in other cancers. Many factors contribute to these challenges, including immune specialization of the brain, molecular heterogeneity, and tumor invasiveness. The standard of care (maximal resection followed by adjuvant temozolomide and radiotherapy - the "Stupp regimen") has increased survival modestly, ${ }^{3,4}$ but no strategy has provided substantial benefits. ${ }^{5}$ Numerous factors have been identified as strongly prognostic for OS, including age, performance status, ${ }^{6}$ radiotherapy, ${ }^{7}$ isocitrate dehydrogenase $1(I D H I)$ mutations, ${ }^{8-10}$ and $O^{6}$-methylguanine DNA methyltransferase (MGMT) methylation.11,12 The 2016 WHO classification guidelines divide glioblastoma into $I D H I$ wild type $(90 \%)$ and $I D H I$ mutant $(10 \%)^{13}$ because this mutation has shown strong association with OS. Extent of resection (EOR) has been shown in many studies to have prognostic impact on progression-free survival (PFS) and OS, ${ }^{14-16}$ but controversy exists regarding the amount of clinical benefit and degree of EOR required. ${ }^{15,17}$

Low-field and high-field intraoperative MRI (iMRI) has been used to safely maximize EOR during cranial surgery, principally for gliomas and pituitary adenomas. ${ }^{18-20}$ Retrospective analyses ${ }^{21,22}$ and limited prospective randomized trials ${ }^{22-24}$ have demonstrated improved survival after maximal resection for glioblastoma. However, a Cochrane Review of randomized studies assessing iMRI and other intraoperative adjuncts concluded that these modalities may increase EOR, but the data quality is low and benefits are uncertain. ${ }^{25}$ Similarly, a systematic review of comparison studies in 2011 suggested that there is level 2 evidence (at best) for the benefit of iMRI over conventional (non-iMRI) surgery for glioblastoma. ${ }^{21}$ Larger multicenter studies assessing the impact of iMRI on EOR and survival have been reported in low-grade glioma ${ }^{26}$ but not glioblastoma. Clearly, more investigation is needed on the effectiveness of iMRI for glioblastoma surgery.

For this study, we used data from a multicenter mixed retrospective/prospective registry to evaluate the impact of iMRI on glioblastoma outcomes while controlling for known prognostic factors.

\section{Methods \\ Study Design}

The IMRIS Multicenter iMRI Neurosurgery Database (I-MiND) registry is a REDCap (Research Electronic Data Capture ${ }^{27}$ registry of patients who have undergone neurosurgical procedures with or without iMRI. Data were col- lected retrospectively back to 1996 and have been accrued prospectively since 2008. Data include preoperative (demographics, comorbidities, and prior treatments); perioperative (surgical, intraoperative imaging, and pathological details); and postoperative (EOR, complications, tumor progression, recurrence, and OS) characteristics. Eleven institutions have entered more than 9000 cases in total. Institutional IRB approval was received at all participating sites. The I-MiND registry was queried for newly diagnosed supratentorial WHO grade IV glioblastoma that underwent resection. Two patient cohorts were created: 1) a "multicenter cohort" that included all cases that were surgically treated over the available time frame (1997-2019; $\mathrm{n}=640,10$ centers); and 2) an "MRI analysis" cohort (n $=286$ ) that included resection cases (>50\% EOR) from the corresponding author's institution performed between 2008 and 2018 with preoperative, intraoperative (for iMRI cases), and postoperative DICOM availability.

\section{Intraoperative MRI}

Intracranial resections were performed with standard techniques until maximal safe resection was completed. Intraoperative imaging was performed using a movable 1.5-T or 3.0-T iMRI device (IMRIS, Inc.) depending on the institution's scanner type. ${ }^{19,28}$ The MRI sequences performed were based on surgeon discretion, but typically included T1-weighted imaging with and without intravenous contrast, and T2-weighted, FLAIR, and diffusion-weighted imaging as indicated. Additional resection with iMRI guidance was based on surgeon discretion.

\section{Data Collection}

Preoperative variables included general predictors and direct predictors of EOR. General predictors were obtained from the multicenter cohort, including age at surgery (years), American Society of Anesthesiologists (ASA) score, and year of surgery. The American Society of Anesthesiologists Physical Status Classification (ASA scores, grouped as ASA I-II or III-V) was used as a surrogate for Karnofsky Performance Scale (KPS) score because ASA scores were more available..$^{29,30}$ The ASA score was captured in 407/640 (63.6\%) cases based on availability in the medical record. Direct predictors of EOR were obtained from the volumetric analysis cohort and included MRI features (bilateral tumor location, eloquent brain involvement, presence of contrast enhancement, multifocal disease, preoperative tumor volume [in $\left.\mathrm{cm}^{3}\right]$, large tumor size $\left[>50 \mathrm{~cm}^{3}\right]$, and ventricle involvement); intended EOR (gross-total resection [GTR] vs subtotal resection [STR]); and primary neurosurgeon. MRI features were obtained from MRI report review, image sequence review, and tu- 
mor volume segmentation (described below). Cases were coded as having eloquent brain location based on MRI review, with tumor in or adjacent to function locations (i.e., motor, sensory, visual, language, basal ganglia). Intended EOR was interpreted from the operative note, not prospectively collected.

Perioperative and/or postoperative variables were obtained from the multicenter cohort and included EOR (GTR vs STR), IDHI mutation status, iMRI use, MGMT methylation status, new permanent neurological deficits (nPND), Stupp therapy (temozolomide and fractionated radiation therapy), and clinical trial participation. EOR was measured in terms of contrast-enhancing (gadolinium) tumor volume. T2-weighted images were used in cases of nonenhancing tumor. IDHI mutation status was available in 356/640 cases (55.6\%) and MGMT methylation status was available in $412 / 640$ cases $(64.4 \%)$, based on date of surgery. Patients who participated in clinical trials or who received investigational agents were grouped together (agents included etoposide, bevacizumab, rindopepimut, NovoCure devices, DCVax, and others). The volumetric analysis cohort was assessed for intraoperative and postoperative EOR by using 3 measures: 1) GTR versus STR for all 640 cases; 2) percent EOR for the 286 cases with volumetric analyses; and 3) residual contrast-enhancing tumor volume (in cubic centimeters $\left[\mathrm{cm}^{3}\right]$ ). Unintended missed assessable residual was recorded when residual tumor was identified on a postoperative scan despite an operative note indicating intended GTR.

\section{Tumor Volumetric Analysis}

Manual tumor segmentation of pre-, intra-, and postoperative MRI sequences was performed on the volumetric analysis cohort of 286 cases by using 3D Slicer version 4.10.2 r28257. ${ }^{31}$ Contrast-enhancing tumor was segmented, except for nonenhancing tumors, for which T2 hyperintensity was segmented. Representative axial slices were segmented and the "fill between slices" tool was used for volume interpolation. Tumor volumes were measured in cubic centimeters $\left(\mathrm{cm}^{3}\right)$. Figure 1 demonstrates an exemplar case showing contrast-enhancing tumor, manual segmentation, and 3D-reconstructed tumor volume at pre-, intra-, and postoperative time points.

\section{Statistical Analysis}

Statistical analyses were performed using IBM SPSS Statistics version 26 (IBM Corp.). A significance threshold of $p \leq 0.05$ was used in all analyses. Chi-square analysis and Student's t-test were used for categorical and continuous variable comparisons, respectively. OS was defined as time (in months) from operation to death (or last known alive date). The median survival times were calculated using the Kaplan-Meier method. Kaplan-Meier analysis was used to assess impact of EOR and iMRI on OS. EOR was categorized (into 100\%, 95\%-99\%, 80\%-94\%, and $50 \%-79 \%$ groupings) to allow for Kaplan-Meier evaluation. For the volumetric analysis cohort of 286 cases, EOR category cutoffs were decided empirically to make reasonable groupings of case counts for evaluation. Univariate and multivariate Cox models were used to assess the im-
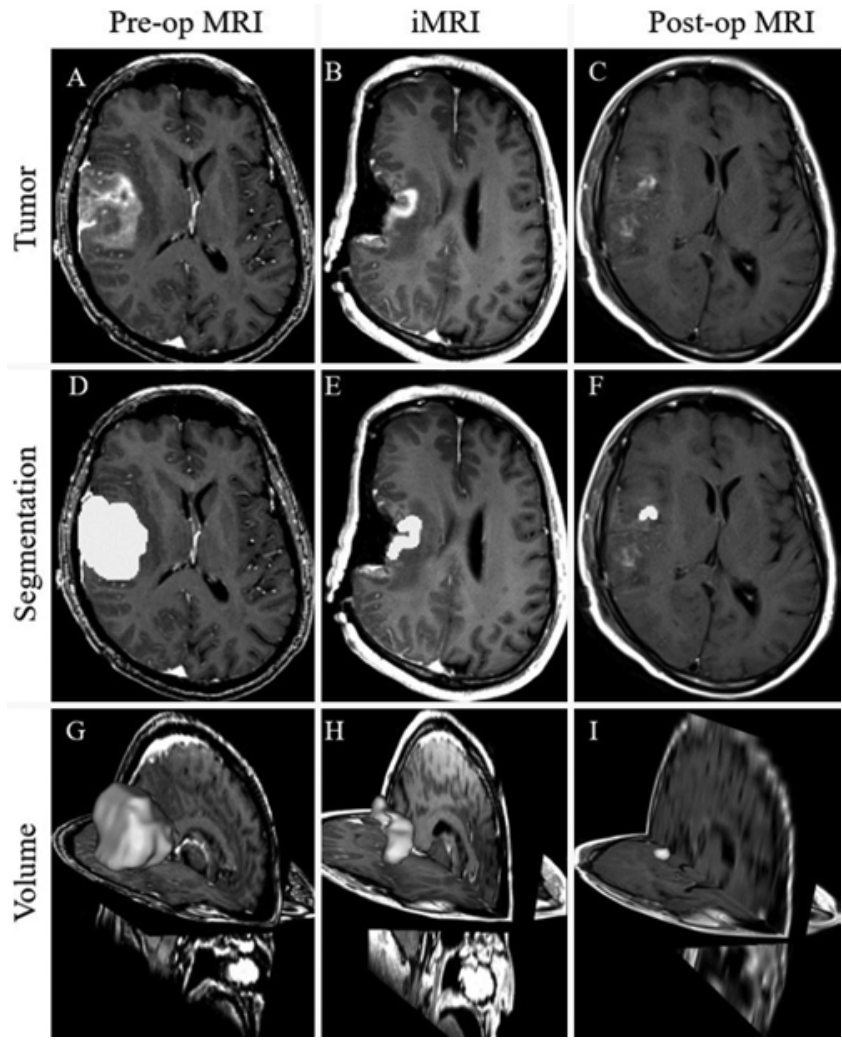

FIG. 1. Case demonstration. Sixty-year-old man with newly diagnosed glioblastoma. Representative contrast-enhanced T1-weighted MR images showing tumor $(\mathbf{A}-\mathbf{C})$, manual segmentations (D-F), and reconstructed tumor volumes $(G-I)$ at preoperative $(A, D$, and $G)$, intraoperative $(B$, $\mathrm{E}$, and $\mathrm{H}$ ), and postoperative (C, F, and I) time points. Preoperative MRI demonstrated an enhancing right temporal parietal tumor measured as $70.7 \mathrm{~cm}^{3}$ by segmentation using 3D Slicer. iMRI demonstrated residual tumor measuring $6.8 \mathrm{~cm}^{3}$. Postoperative MRI demonstrated residual tumor volume of $0.5 \mathrm{~cm}^{3}$. iMRI therefore impacted a reduction of residual tumor by $6.3 \mathrm{~cm}^{3}$ ( $8.9 \%$ of resected tumor volume), with an ultimate residual enhancing tumor volume of $0.7 \%$ of the original tumor volume.

pact of variables on OS. Univariate and multivariate binary logistic regression was performed to assess the impact of variables on EOR. Significant predictors on univariate analysis were included in subsequent multivariate analysis. Stepwise backward removal of variables using likelihood ratio analysis was performed to obtain final Cox models of OS. Model outputs were represented as hazard ratios, odds ratios, and 95\% confidence intervals. Patients were excluded listwise in the building of multiple regression models.

\section{Results}

Specific EOR and iMRI analyses were performed using data from the volumetric analysis cohort $(\mathrm{n}=286 ; 110$ non-iMRI, $176 \mathrm{iMRI)}$. The median pre- and postoperative tumor volumes were $34.1 \mathrm{~cm}^{3}$ (range $0.6-163.0 \mathrm{~cm}^{3}$ ) and $0.0 \mathrm{~cm}^{3}$ (range $0.0-37.0 \mathrm{~cm}^{3}$ ), respectively (means 38.7 and $1.4 \mathrm{~cm}^{3}$, respectively). Post-iMRI-guided resection was performed in 104/122 (85.2\%) cases with STR demonstrated on iMRI after initial resection. Percent residual tumor volume on iMRI and first postoperative scan are illustrated in Fig. 2. For iMRI cases with volumetric analysis, 


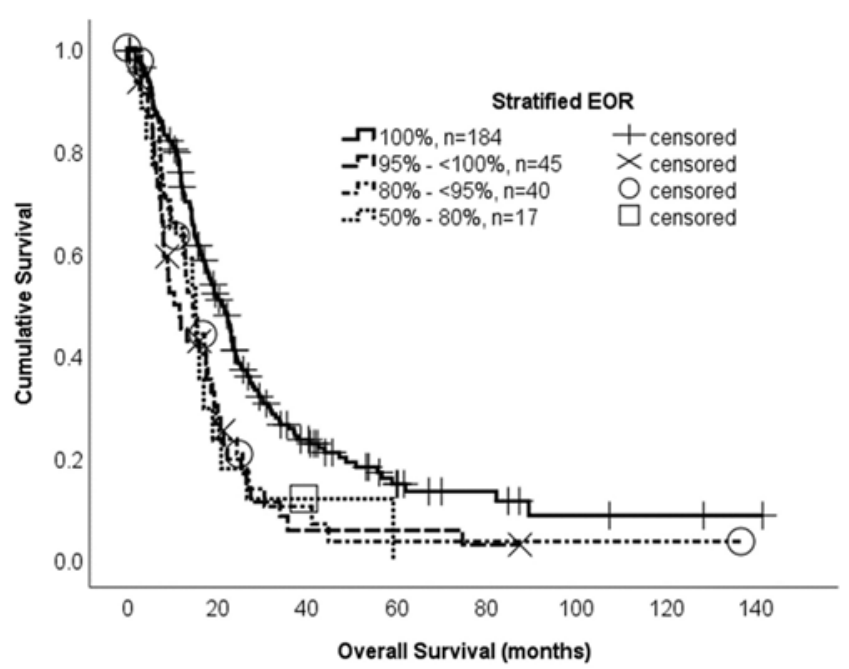

FIG. 2. Kaplan-Meier analysis of OS based on stratified EOR. The volumetric analysis cohort $(n=286)$ was evaluated. Log-rank test, $p<0.01$.

the GTR rate increased from 54/176 (30.7\%) on iMRI to $126 / 176(71.5 \%)$ postoperatively (absolute increase $40.8 \%$ ). Five of $176(2.8 \%)$ cases identified as STR on iMRI were GTR on postoperative scan without additional resection (i.e., iMRI false positive). Additional resection after iMRI resulted in a $6.3 \%$ mean increase in percent EOR (median $2.7 \%$, minimal $-1.9 \%$, maximal $79.9 \%$ ) and a $2.2-\mathrm{cm}^{3}$ mean decrease in tumor volume (median $0.9 \mathrm{~cm}^{3}$, minimal $-1.4 \mathrm{~cm}^{3}$, maximal $48.1 \mathrm{~cm}^{3}$ ).
Tumor volumes and EOR comparisons between iMRI and non-iMRI groups were assessed for the volumetric analysis cohort. Cases were further divided into intended GTR group and intended STR group (Table 1). Preoperative tumor volume was significantly greater for iMRI cases in the intended STR group; otherwise, no differences were noted. No differences in postoperative tumor volume were observed. Percent EOR was significantly higher with iMRI for both intended GTR and intended STR. iMRI cases had a significantly higher GTR rate for both intended GTR and intended STR cases. No differences in missed assessable residual tumor were observed between the intended GTR and intended STR cases. Kaplan-Meier analysis of percent EOR (stratified into 100\%, 95\%-99\%, $80 \%-94 \%$, and $50 \%-79 \%$ groupings) showed longer OS for $100 \%$ EOR (GTR) compared to all other groups (logrank test, p < 0.01; Fig. 3); therefore, GTR was used as a covariate in subsequent OS analyses instead of EOR.

Univariate and multivariate modeling was performed using the available data from both the total multicenter (n $=640)$ and volumetric analysis $(n=286)$ cohorts. The median age was 60.0 years (mean 58.5 years, range 20.5-86.3 years). An iMRI scan was performed in 332/640 cases $(51.9 \%)$. Univariate features associated with iMRI use included primary neurosurgeon, year of surgery, tumor size $>50 \mathrm{~cm}^{3}$, EOR, Stupp therapy, and clinical trial participation (Table 2). GTR was achieved in 403/640 total cases (63.0\%). Univariate features associated with STR included bilateral tumor location, eloquent brain involvement, intended STR, multifocal disease, ventricular involvement, no iMRI use, no nPND after surgery, no Stupp therapy,

TABLE 1. Tumor volume and EOR outcomes for the total, intended GTR, and intended STR groups

\begin{tabular}{|c|c|c|c|c|c|c|c|c|c|}
\hline \multirow[b]{2}{*}{ Variable } & \multicolumn{3}{|c|}{ Total } & \multicolumn{3}{|c|}{ Intended GTR } & \multicolumn{3}{|c|}{ Intended STR } \\
\hline & Non-iMRI & iMRI & p Value & Non-iMRI & iMRI & $p$ Value & Non-iMRI & iMRI & $p$ Value \\
\hline Counts & 110 & 176 & & 80 & 138 & & 30 & 38 & \\
\hline \multicolumn{10}{|l|}{ Preop vol, $\mathrm{cm}^{3}$} \\
\hline Mean & 35.6 & 40.7 & 0.15 & 36.2 & 38.0 & 0.66 & 34.0 & 50.5 & 0.02 \\
\hline Median & 29.0 & 37.0 & & 28.5 & 35.0 & & 29.0 & 44.1 & \\
\hline Range & $1.8-163.0$ & $0.6-134.0$ & & $1.8-127.1$ & $0.6-134.0$ & & $4.5-162.9$ & $6.4-121.0$ & \\
\hline \multicolumn{10}{|l|}{ Intraop vol, $\mathrm{cm}^{3}$} \\
\hline Mean & NA & 3.1 & NA & NA & 1.8 & NA & NA & 7.8 & NA \\
\hline Median & NA & 0.6 & & NA & 0.3 & & & 4.0 & \\
\hline Range & NA & $0.0-49.7$ & & NA & $0.0-48.1$ & & & $0-49.7$ & \\
\hline \multicolumn{10}{|l|}{ Postop vol, $\mathrm{cm}^{3}$} \\
\hline Mean & 1.8 & 1.2 & 0.15 & 0.5 & 0.3 & 0.17 & 5.3 & 4.3 & 0.55 \\
\hline Median & 0.0 & 0.0 & & 0.0 & 0.0 & & 3.0 & 2.0 & \\
\hline Range & $0.0-26.3$ & $0.0-37.0$ & & $0.0-10.1$ & $0.0-8.4$ & & $0.3-26.3$ & $0.0-37.0$ & \\
\hline \multicolumn{10}{|l|}{ EOR, \% } \\
\hline Mean & 93.8 & 97.6 & $<0.01$ & 97.7 & 99.2 & 0.02 & 83.4 & 91.7 & $<0.01$ \\
\hline Median & 100 & 100 & & 100 & 100 & & 85.2 & 96.0 & \\
\hline Range & $51.5-100$ & $64.0-100$ & & 59.5-100 & $67.8-100$ & & $51.5-96.7$ & $64.0-100$ & \\
\hline Postop GTR, no. (\%) & $58(52.7)$ & $126(71.5)$ & $<0.01$ & $58(72.5)$ & $115(83.3)$ & 0.06 & $0(0.0)$ & $11(28.9)$ & $<0.01$ \\
\hline Missed assessable residual tumor, no. (\%) & $27(24.5)$ & $30(17)$ & 0.12 & $21(26.3)$ & $22(15.9)$ & 0.07 & $6(20.0)$ & $8(21.1)$ & 0.92 \\
\hline
\end{tabular}

NA = not applicable.

Volumetric analysis cohort $(n=286)$ was evaluated. Boldface type indicates statistical significance. 


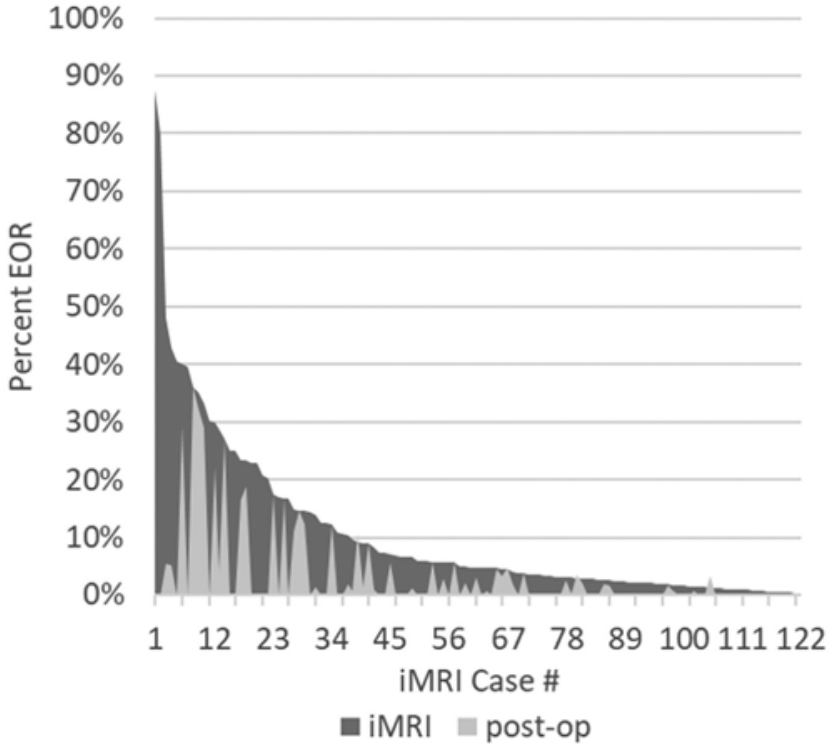

FIG. 3. Percent EOR after iMRI (dark gray) and postoperative MRI (light gray) for all 122 cases with STR on iMRI. Percent EOR represented the change in percent tumor volume compared to the preoperative tumor volume. Cases were ordered from lowest to highest percent EOR on iMRI. The volumetric analysis cohort $(n=286)$ was evaluated. and no clinical trial participation (Table 2). Multivariate features associated with STR included no iMRI use and intended STR (Table 3). The median OS for the multicenter cohort was 17.0 months (95\% CI 15.6-18.4 months). Univariate features associated with shorter OS included older age, ASA score > II, eloquent brain involvement, intended STR, multifocal disease, STR, IDH1 wild type, iMRI use, no MGMT methylation, no Stupp therapy, and no clinical trial participation (Table 2). Multivariate features associated with shorter OS included old age, STR, IDH1 wild type, no MGMT methylation, and no Stupp therapy (Table 4). iMRI was not a significant predictor of OS on multivariate analyses when including EOR as a predictor or after EOR was removed (Table 4).

\section{Discussion}

This multicenter experience with iMRI-guided resection of adult newly diagnosed supratentorial glioblastoma details the efficacy of $\mathrm{iMRI}$ in improving EOR and provides detailed risk-adjusted outcome assessments of EOR and OS. Univariate and multivariate analyses demonstrated improved EOR using iMRI compared to conventional surgery. Increased EOR was associated with longer OS

TABLE 2. Preoperative, perioperative, and postoperative variables related to iMRI use, EOR (GTR vs STR), and OS

\begin{tabular}{|c|c|c|c|c|c|c|c|}
\hline \multirow[b]{2}{*}{ Variable } & \multirow[b]{2}{*}{ No. } & \multicolumn{2}{|l|}{ iMRI } & \multicolumn{2}{|l|}{ EOR } & \multicolumn{2}{|l|}{ OS } \\
\hline & & OR $(95 \% \mathrm{Cl})$ & p Value & OR $(95 \% \mathrm{Cl})$ & p Value & $\mathrm{HR}(95 \% \mathrm{Cl})$ & $\mathrm{p}$ Value \\
\hline \multicolumn{8}{|l|}{ Preop variables } \\
\hline \multicolumn{8}{|c|}{ Multicenter cohort data } \\
\hline Age & 640 & $0.99(0.97-1.00)$ & 0.12 & $1.00(0.99-1.01)$ & 0.46 & $1.02(1.00-1.03)$ & $<0.01$ \\
\hline ASA score >II & $229 / 406$ & $1.10(0.74-1.63)$ & 0.62 & $0.99(0.66-1.48)$ & 0.98 & $1.32(1.05-1.65)$ & 0.02 \\
\hline Surgery yr* & 640 & NA & $<0.01$ & NA & 0.11 & NA & 0.11 \\
\hline \multicolumn{8}{|c|}{ Volumetric analysis cohort data } \\
\hline Bilat & $19 / 286$ & $2.46(0.79-7.64)$ & 0.12 & $3.37(1.28-8.85)$ & 0.01 & $1.15(0.67-1.98)$ & 0.60 \\
\hline Eloquent & $50 / 286$ & $1.13(0.60-2.14)$ & 0.69 & 9.73 (4.69-20.17) & $<0.01$ & $1.62(1.16-2.26)$ & $<0.01$ \\
\hline Enhancing & $280 / 286$ & $0.31(0.03-2.72)$ & 0.29 & NA & 0.99 & $1.42(0.52-3.84)$ & 0.48 \\
\hline Intended STR & $68 / 286$ & $0.73(0.42-1.27)$ & 0.27 & $19.92(9.65-41.09)$ & $<0.01$ & $1.73(1.28-2.33)$ & $<0.01$ \\
\hline Multifocal & $33 / 286$ & $0.72(0.34-1.49)$ & 0.38 & $7.14(3.08-16.54)$ & $<0.01$ & $1.98(1.35-2.90)$ & $<0.01$ \\
\hline Neurosurgeon* & 286 & NA & $<0.01$ & NA & 0.09 & NA & 0.75 \\
\hline Preop vol & 286 & $1.00(0.99-1.01)$ & 0.15 & $1.00(0.99-1.01)$ & 0.34 & $1.00(0.99-1.01)$ & 0.33 \\
\hline Tumor $>50 \mathrm{~cm}^{3}$ & $144 / 286$ & $1.74(1.08-2.83)$ & 0.02 & $1.41(0.86-2.29)$ & 0.16 & $1.27(0.98-1.65)$ & 0.07 \\
\hline Ventricle & $71 / 286$ & $1.20(0.68-2.10)$ & 0.52 & $2.51(1.45-4.34)$ & $<0.01$ & $1.33(0.98-1.80)$ & 0.06 \\
\hline \multicolumn{8}{|l|}{ Peri-/postop variables } \\
\hline EOR-STR & $237 / 640$ & $1.71(1.24-2.36)$ & $<0.01$ & NA & NA & $1.55(1.28-1.86)$ & $<0.01$ \\
\hline IDH1 mutated & $33 / 356$ & $1.54(0.64-3.67)$ & 0.33 & $1.44(0.66-3.14)$ & 0.35 & $0.34(0.19-0.61)$ & $<0.01$ \\
\hline iMRI & $335 / 640$ & NA & NA & $0.58(0.42-0.81)$ & $<0.01$ & $0.82(0.69-0.98)$ & 0.03 \\
\hline MGMT methylated & $160 / 412$ & $1.40(0.91-2.16)$ & 0.12 & $0.87(0.58-1.30)$ & 0.52 & $0.65(0.51-0.83)$ & $<0.01$ \\
\hline nPND & $57 / 640$ & $1.51(0.87-2.61)$ & 0.14 & $0.53(0.31-0.90)$ & 0.02 & $1.08(0.80-1.47)$ & 0.61 \\
\hline Stupp therapy & $437 / 640$ & $2.44(1.74-3.42)$ & $<0.01$ & $0.49(0.34-0.71)$ & $<0.01$ & $0.46(0.38-0.56)$ & $<0.01$ \\
\hline Trial & $141 / 640$ & $2.34(1.58-3.47)$ & $<0.01$ & $0.50(0.34-0.73)$ & $<0.01$ & $0.59(0.47-0.75)$ & $<0.01$ \\
\hline
\end{tabular}

General predictors were obtained from the multicenter cohort $(n=640)$, whereas predictors of EOR were obtained from the volumetric analysis cohort $(n=286)$. Logistic regression models were produced to evaluate univariate prediction of IMRI and EOR. Cox proportional hazards models were produced to evaluate univariate prediction of OS. Boldface type indicates statistical significance.

* Odds ratios not listed for nonsignificant multilevel categorical variables. 
TABLE 3. Multivariate logistic regression models of STR

\begin{tabular}{lcc}
\hline \multirow{2}{*}{ Variable } & \multicolumn{2}{c}{ Multivariate } \\
\cline { 2 - 3 } & OR $(95 \% \mathrm{Cl})$ & p Value \\
\hline Bilat & $1.00(0.25-4.00)$ & 0.99 \\
\hline Eloquent & $1.45(0.44-4.78)$ & 0.54 \\
\hline iMRI & $0.35(0.18-0.66)$ & $<0.01$ \\
\hline Intended STR & $13.71(4.6-40.88)$ & $<0.01$ \\
\hline Multifocal & $1.50(0.49-4.59)$ & 0.47 \\
\hline Stupp therapy complete & $0.85(0.43-1.68)$ & 0.65 \\
\hline Ventricle & $1.96(0.93-4.14)$ & 0.07 \\
\hline
\end{tabular}

Volumetric analysis cohort $(n=286)$ evaluated. Boldface type indicates statistical significance.

in univariate and multivariate modeling, and iMRI use was associated with longer OS in univariate (although not multivariate) modeling. In aggregate, this study supports the use of iMRI for primary glioblastoma; however, more work is needed to understand the true clinical impact of this technology.

The intention when using iMRI is to safely increase tumor removal, which was observed in this study. The percent EOR and the GTR rate were higher in the iMRI group, including in the intended GTR and intended STR subgroups (Table 3). Some controversy exists regarding the importance of EOR for glioblastoma surgery. A 2011 study by Sanai et al. of 500 consecutive newly diagnosed patients with supratentorial glioblastoma suggested that EOR greater than $78 \%$ was sufficient for improved OS, with progressive increases in EOR associated with longer OS. ${ }^{14} \mathrm{~A}$ larger $(\mathrm{n}=27,865)$ but less granular National Cancer Database study by Trifiletti et al. in 2017 showed a significant difference between GTR and biopsy (HR 0.768, 95\% CI $0.683-0.863 ; p<0.001)$, but no difference in OS between STR and biopsy (HR 0.995, 95\% CI 0.888-1.115; $\mathrm{p}=0.930) .32$

This current study used manual segmentation to volumetrically evaluate the impact of iMRI on EOR and the effect of EOR on OS. Kuhnt et al. ${ }^{33}$ reported on 135 primary and recurrent glioblastoma resections performed with 1.5$\mathrm{T}$ iMRI, finding that the GTR rate increased from $34.8 \%$ to $41.5 \%$ after $\mathrm{iMRI}$, and that age $\geq 65$ years (HR 0.61 , 95\% CI $0.38-0.97 ; \mathrm{p}<0.05$ ) and EOR $\geq 98 \%$ (HR 0.39, $95 \%$ CI $0.24-0.63 ; \mathrm{p}=0.001$ ) were significant predictors of OS in multivariate modeling. A study by Coburger et al. suggested that iMRI has benefits for EOR and OS even in cases with intended STR. ${ }^{34}$

Kaplan-Meier analysis showed that GTR was associated with increased OS when compared independently to other stratified EOR groupings (i.e., 50\%-79\%, 80\%-94\%, and 95\%-99\% EOR; Fig. 3). Due to these findings, GTR was used as the dependent variable in subsequent regression modeling. Univariate logistic regression models showed several significant predictors of GTR (bilateral location, eloquent brain involvement, iMRI, intended GTR, multifocal disease, Stupp therapy, and ventricular involvement); however, on multivariate evaluation only intended GTR and iMRI remained significant (Table 4). Minimal controversy exists regarding the importance of GTR on OS for glioblastoma. Several studies report OS benefit from GTR, although this is not supported by high-quality evidence..$^{25}$ A large series of 1229 patients with glioblastoma from Li et al. showed increased OS with GTR (100\% EOR) compared to STR $(78 \%$ to $<100 \%)$ on multivariate analysis (HR 1.53, 95\% CI 1.33-1.77; $\mathrm{p}<0.001) .{ }^{16}$ Additional multivariate predictors of longer OS included younger age, higher KPS score, cyst on imaging, no prior treatment, and smaller preoperative contrast-enhancing tumor volume. Prior treatment and $<53.21 \%$ resection of supramarginal T2/FLAIR hyperintensity were associated with decreased OS.

Univariate modeling showed several significant predictors of OS (age, ASA score > II, eloquent brain involvement, intended EOR, multifocal disease, EOR, IDHI mutation status, iMRI use, MGMT methylation, Stupp therapy completion, and clinical trial participation); however, on multivariate testing, only age, EOR, Stupp therapy, IDHI mutation, and MGMT methylation remained significant. These results are in line with prior reports. Several notable studies have used multivariate techniques to evaluate predictors of OS after surgery for glioblastoma. Lacroix et al. evaluated 416 consecutive patients after surgery for glioblastoma and reported multivariate predictors of OS to include KPS score, age, EOR, amount of necrosis, and degree of enhancement. ${ }^{6}$ Pan et al. ${ }^{2}$ reviewed 14,675 glioblastoma patients in the Surveillance, Epidemiology, and End Results (SEER) data set and found that multivariate predictors of OS included age, male sex, unmarried relationship status, white race, non-northeast US residential status, no adjuvant therapy, and EOR. Trifiletti et al. analyzed 27,865 patients from the National Cancer Database and showed that increased OS was associated with younger age, female sex, race, fewer comorbidities, higher KPS score, smaller tumor, unifocal tumor, MGMT methylation, Stupp therapy, and facility volume..$^{32}$ A retrospective study of 115 patients with glioblastoma performed by Rahman et al. showed a significant association between nPND and OS, but this was not observed in the current study. ${ }^{35}$

In the current study, iMRI was a significant predictor of OS in univariate but not in multivariate modeling. Results from prior studies comparing OS between iMRI and conventional surgery have been mixed. A systematic review by Kubben et al. in 2011 identified 12 nonrandomized studies, discussed the numerous limitations, and stated that level 2 evidence (at best) exists regarding the impact of iMRI on EOR, with less certainty regarding OS. ${ }^{21} \mathrm{~A}$ meta-analysis published in 2017 by Li et al. evaluated 8 studies comparing iMRI to conventional surgery. ${ }^{22}$ iMRI was associated with a greater rate of GTR (OR 3.16, 95\% CI 2.07-4.83; $\mathrm{p}<0.001)$ and a higher of PFS (OR 1.84, 95\% CI 1.15-2.95; $\mathrm{p}=0.012)$, but no difference in OS ( $\mathrm{p}=$ 0.799). By mechanism, the apparent GTR benefit provided by iMRI should translate to increased OS but is probably difficult to demonstrate and/or quantify given the high heterogeneity of disease (with multiple predictors with strong influence on outcome).

\section{Study Justification}

There are several important reasons to understand the true impact of iMRI on glioblastoma resection. First, there 
TABLE 4. Multivariate Cox models of OS

\begin{tabular}{lcrrrr}
\hline & \multicolumn{2}{c}{ Model 1} & & \multicolumn{2}{c}{ Model 2 } \\
\cline { 2 - 3 } \cline { 5 - 6 } Variable & $\mathrm{HR}(95 \% \mathrm{Cl})$ & $\mathrm{p}$ Value & & $\mathrm{HR}(95 \% \mathrm{Cl})$ & $\mathrm{p}$ Value \\
\hline Significant univariate predictors & & & & \\
\hline Age & $1.02(1.01-1.04)$ & $<0.01$ & & $1.03(1.01-1.04)$ & $<0.01$ \\
\hline EOR-GTR & $0.54(0.40-0.73)$ & $<0.01$ & & $\mathrm{NA}$ & $\mathrm{NA}$ \\
\hline IDH1 mutated & $0.43(0.22-0.83)$ & $\mathbf{0 . 0 1}$ & & $0.51(0.26-0.97)$ & 0.04 \\
\hline MGMT methylated & $0.57(0.43-0.76)$ & $<0.01$ & & $0.60(0.45-0.81)$ & $<0.01$ \\
\hline Stupp therapy & $0.40(0.29-0.54)$ & $<0.01$ & & $0.40(0.30-0.55)$ & $<0.01$ \\
\hline iMRI $^{*}$ & $0.92(0.67-1.24)$ & 0.59 & & $0.80(0.59-1.08)$ & 0.16 \\
\hline
\end{tabular}

The models include 324 patients with 208 events. General pre-, peri-, and postoperative predictors of OS on univariate analysis were selected for model entry using the stepwise backward likelihood ratio method. Model 1 includes all predictors; model 2 has EOR removed. Boldface type indicates statistical significance.

${ }^{*}$ iMRI was included despite lack of significance.

is controversy among neurosurgeons regarding the benefit of this technology. The present study and prior literature give support for the benefit of iMRI for glioblastoma surgery, but the quantitative impact of iMRI is not well established. Jenkinson et al. in 2018 presented a Cochrane Review identifying 4 randomized controlled trials evaluating intraoperative imaging technology, including 2 trials involving iMRI (Senft et al., 2011, $\mathrm{n}=58$; Kubben et al., 2014, $\mathrm{n}=14) .{ }^{23-25}$ The report concluded that available MRI trials offered only low-quality to very low-quality evidence for the EOR maximization for high-grade gliomas, and that the clinical benefits for PFS, OS, and quality of life are uncertain. Higher-quality evidence (randomized clinical trials, large patient numbers, more sophisticated models, and multicenter efforts) are needed.

Additionally, iMRI is now competing with other technologies-most notably 5-aminolevulinic acid (5-ALA), which has recently been approved by the FDA for use in US medical institutions. In 2006, Stummer et al. provided interim analysis of a randomized controlled trial containing 270 patients, showing that 5-ALA increased the rate of GTR by $29 \%(\mathrm{p}<0.0001)$ and increased 6 -month PFS by $19.9 \%(p=0.0003)$ without toxic effect. ${ }^{36}$ No significant difference was noted in OS, but the authors stated that the study was not specifically powered to evaluate this outcome. A small study $(n=117)$ by Roder et al. suggested a mean residual tumor volume advantage of iMRI $(n=27$, $\left.0.5[0.0-4.7] \mathrm{cm}^{3}\right)$ versus 5-ALA $(\mathrm{n}=47,1.9[0.0-13.2]$ $\mathrm{cm}^{3}$ ), but this study was small in size and susceptible to allocation bias without controlling for predictors of EOR. ${ }^{37}$ Coburger et al. suggested that 5-ALA may be a complementary tool to iMRI, in 1 small study showing significantly increased EOR with iMRI + 5-ALA (100\%) compared to iMRI alone $(82 \%, \mathrm{p}<0.01){ }^{38}$

Furthermore, there remains controversy regarding the cost-effectiveness of iMRI, especially in the setting of less expensive technologies like intraoperative ultrasound (iUS) ${ }^{39}$ iMRI requires ample investment in terms of upfront cost, maintenance, operative time, and surgical team effort. Few cost-effectiveness studies for iMRI exist. Eljamel and Mahboob in 2016 compared the costs of several intraoperative adjuvants used in glioblastoma resection (5-ALA, fluorescein, iUS, and iMRI) using data from a literature review..$^{40}$ Cost included additional capital cost $[$ ACC $=$ purchase $\operatorname{cost} /($ depreciation $\times$ number of cases per year)] multiplied by $20 \%$ because iUS and iMRI were used for other applications (e.g., low-grade glioma resection, pituitary adenoma resection, etc.). Costs associated with longer operative times, image interpretation, scanner operation and maintenance, and downstream treatment effect were not included. Incremental quality-adjusted life years (QALYs) associated with each technology were based on estimated PFS benefit from increased GTR calculated from meta-analysis. Patient heterogeneity, reported PFS, and OS were not included. The incremental costs per QALY were $\$ 16,218, \$ 3181, \$ 6049$, and $\$ 32,954$, respectively, for the 4 adjuvants. Abraham et al. in 2019 presented a more detailed microsimulation cost-effectiveness model of iMRI for glioblastoma resection..$^{41}$ In their study, iMRI added an incremental benefit of 0.18 QALYs (1.34 vs 1.16) at a cost of $\$ 13,447$, for an incremental cost-effectiveness ratio of $\$ 76,442$ per QALY. The authors concluded that iMRI was deemed cost-effective at a reasonable threshold of $\$ 100,000$ per QALY. The major limitation was that model probabilities (e.g., GTR, PFS, and OS) were based on the limited studies currently available.

Large national databases and patient registries are effective tools for overcoming some of the key limitations in neurosurgical research, including small sample sizes, selection bias, and relative lack of high-quality randomized clinical trials. Efforts such as the National Neurosurgery Quality Outcomes Database $\left(\mathrm{N}^{2} \mathrm{QOD}\right)^{42}$ have created detailed multivariate models to adjust for bias and to control for influential confounders in the assessment of patient outcomes. The I-MiND registry was in large part created to assess the patient-level value provided by iMRI for different intracranial pathologies. In the absence of large randomized controlled trials, multicenter repositories like the I-MiND registry may represent the most effective tool for creating robust data sets for evaluation in efficacy studies and may provide inputs for modeling the cost-effectiveness of iMRI for glioblastoma and other intracranial pathologies.

\section{Limitations}

The results were collected prospectively, but analyses 
were retrospective. Factors impacting glioblastoma outcomes are multifactorial. Multivariate modeling was used, but the possibility of uncontrolled confounding cannot be excluded. In the present study, MRI features including bilateral tumor location, eloquent brain involvement, contrast enhancement, multifocal disease, and ventricle involvement were evaluated. Prior studies have evaluated MRI predictors of OS not captured in the present study. Henker et al. reported on 114 patients with glioblastoma, evaluating the impact of tumor necrosis and edema volume, and found the necrosis/tumor ratio to be significantly associated with OS in multivariate analysis (HR 2.63; p $=0.016) .{ }^{43}$ A study by Zhang et al. of 147 patients with primary glioblastoma showed that a higher ratio of perilesional edema to contrast-enhancing tumor was significantly associated with OS. ${ }^{44}$ Factors such as amount of necrosis, ${ }^{6}$ degree of enhancement, ${ }^{6}$ and cyst on imaging ${ }^{16}$ have been found to be associated with OS in prior literature and were not recorded in this study.

In the present study, EOR was determined by segmentation of residual enhancement (or T2 hyperintensity for noncontrast-enhancing tumors), but some evidence suggests that "supramaximal" resection of perilesional FLAIR abnormalities may improve OS and PFS, which was not captured in the present study. ${ }^{916}$ Beiko et al.'s report from 2014 on 207 glioblastoma patients suggested that supramaximal resection improved OS specifically for patients with $I D H I$ mutated tumors. ${ }^{9}$ A more recent study from Molinaro et al. in 2020 evaluated 761 patients and found an association between supramaximal resection and longer OS for younger patients (regardless of IDHI status) and patients with $I D H I$ wild-type tumors. ${ }^{45}$

Moreover, iMRI and non-iMRI groups were not randomly allocated; therefore, results are susceptible to allocation bias. Preoperative predictors of group assignment included the primary neurosurgeon, surgery year, and tumor size $>50 \mathrm{~cm}^{3}$. Case matching was not performed because predictors of iMRI group assignment were not associated with EOR or OS. In our experience, surgeons may elect not to perform an iMRI scan if they are confident with the EOR, which would result in uncontrolled selection bias of both GTR and STR cases with unclear impact on outcomes. The results of EOR after iMRI may be biased. Based on our experience, less aggressive resection was pursued in at least some iMRI cases because surgeons had another chance for resection after the iMRI scan. Figure 2 probably represents an overrepresentation of the benefit of iMRI for EOR. Incomplete data were available for IDH1 mutation and MGMT methylation status, but missing genetic data were directly related to the date of surgery and should not specifically bias OS results. The ASA score, which poorly captures functional status, was used as a measure of preoperative performance status (although a few studies have shown it to be a modest surrogate for KPS score).

The present study is a preliminary effort that includes data from a multicenter cohort and volumetric analysis cohort from a single center. DICOM images and medical records are currently not shared between institutions, but this is an area of great interest for this research team. Future studies should involve complete multicenter data, which will increase statistical power and reduce institutional bias. Evaluation of GTR was challenging in some cases due to postoperative enhancement or contrast leak, and this bias probably increased the number of STR cases. This bias is amplified in the present study, because GTR was used as a variable/outcome measure instead of the percent EOR (given strong GTR associated with improved OS; see Fig. 3). This bias was not specific to iMRI versus non-iMRI grouping and is probably present in most prior iMRI research.

\section{Conclusions}

As with prior works, the present study demonstrates a strong association between GTR and OS for primary glioblastoma. EOR and GTR were greater with iMRIguided surgery compared to conventional surgery, without a significant increase in neurological complications. The increase in EOR and GTR from iMRI use was not translated to OS after controlling for other prognostic factors. These findings suggest that iMRI has a role in improving outcomes for patients with glioblastoma, but more study is required. Future registry studies should include higher case numbers, more complete data sets, and better control of heterogeneity.

\section{Acknowledgments}

We thank Dr. Yan Yan for his statistical guidance; Evan Amann for assistance with data collection; Dan Marcus and the Neuroinformatics Research Group (NRG) for assistance with image retrieval and archive; Nicolas G. Chicoine for assistance with image processing and tumor segmentation; and the Departments of Neurosurgery at participating institutions for their support.

\section{References}

1. Ostrom QT, Gittleman H, Liao P, et al. CBTRUS statistical report: primary brain and central nervous system tumors diagnosed in the United States in 2007-2011. Neuro Oncol. 2014;16(suppl 4):iv1-iv63.

2. Pan IW, Ferguson SD, Lam S. Patient and treatment factors associated with survival among adult glioblastoma patients: a USA population-based study from 2000-2010. J Clin Neurosci. 2015;22(10):1575-1581.

3. Stupp R, Hegi ME, Mason WP, et al. Effects of radiotherapy with concomitant and adjuvant temozolomide versus radiotherapy alone on survival in glioblastoma in a randomised phase III study: 5-year analysis of the EORTC-NCIC trial. Lancet Oncol. 2009;10(5):459-466.

4. Stupp R, Mason WP, van den Bent MJ, et al. Radiotherapy plus concomitant and adjuvant temozolomide for glioblastoma. N Engl J Med. 2005;352(10):987-996.

5. Woehrer A, Bauchet L, Barnholtz-Sloan JS. Glioblastoma survival: has it improved? Evidence from population-based studies. Curr Opin Neurol. 2014;27(6):666-674.

6. Lacroix M, Abi-Said D, Fourney DR, et al. A multivariate analysis of 416 patients with glioblastoma multiforme: prognosis, extent of resection, and survival. J Neurosurg. 2001; 95(2):190-198.

7. Mineo JF, Bordron A, Baroncini M, et al. Prognosis factors of survival time in patients with glioblastoma multiforme: a multivariate analysis of 340 patients. Acta Neurochir (Wien). 2007;149(3):245-253.

8. Yan H, Parsons DW, Jin G, et al. IDH1 and IDH2 mutations in gliomas. N Engl J Med. 2009;360(8):765-773. 
9. Beiko J, Suki D, Hess KR, et al. IDH1 mutant malignant astrocytomas are more amenable to surgical resection and have a survival benefit associated with maximal surgical resection. Neuro Oncol. 2014;16(1):81-91.

10. Miller JJ, Shih HA, Andronesi OC, Cahill DP. Isocitrate dehydrogenase-mutant glioma: evolving clinical and therapeutic implications. Cancer. 2017;123(23):4535-4546.

11. Cao VT, Jung TY, Jung S, et al. The correlation and prognostic significance of MGMT promoter methylation and MGMT protein in glioblastomas. Neurosurgery. 2009;65(5):866-875.

12. Hegi ME, Diserens AC, Gorlia T, et al. MGMT gene silencing and benefit from temozolomide in glioblastoma. $N$ Engl $J$ Med. 2005;352(10):997-1003.

13. Louis DN, Perry A, Reifenberger G, et al. The 2016 World Health Organization Classification of Tumors of the Central Nervous System: a summary. Acta Neuropathol. 2016;131(6): 803-820.

14. Sanai N, Polley MY, McDermott MW, et al. An extent of resection threshold for newly diagnosed glioblastomas. $J$ Neurosurg. 2011;115(1):3-8.

15. Brown TJ, Brennan MC, Li M, et al. Association of the extent of resection with survival in glioblastoma: a systematic review and meta-analysis. JAMA Oncol. 2016;2(11):1460-1469.

16. Li YM, Suki D, Hess K, Sawaya R. The influence of maximum safe resection of glioblastoma on survival in 1229 patients: Can we do better than gross-total resection? J Neurosurg. 2016;124(4):977-988.

17. Sanai N, Berger MS. Glioma extent of resection and its impact on patient outcome. Neurosurgery. 2008;62(4):753-764, 264-266.

18. Nimsky C, Ganslandt O, Von Keller B, et al. Intraoperative high-field-strength MR imaging: implementation and experience in 200 patients. Radiology. 2004;233(1):67-78.

19. Chicoine MR, Lim CC, Evans JA, et al. Implementation and preliminary clinical experience with the use of ceiling mounted mobile high field intraoperative magnetic resonance imaging between two operating rooms. Acta Neurochir Suppl. 2011;109:97-102.

20. Haydon DH, Chicoine MR, Dacey RG Jr. The impact of highfield-strength intraoperative magnetic resonance imaging on brain tumor management. Neurosurgery. 2013;60(suppl 1): 92-97.

21. Kubben PL, ter Meulen KJ, Schijns OE, et al. Intraoperative MRI-guided resection of glioblastoma multiforme: a systematic review. Lancet Oncol. 2011;12(11):1062-1070.

22. Li P, Qian R, Niu C, Fu X. Impact of intraoperative MRIguided resection on resection and survival in patient with gliomas: a meta-analysis. Curr Med Res Opin. 2017;33(4): 621-630.

23. Senft C, Bink A, Franz K, et al. Intraoperative MRI guidance and extent of resection in glioma surgery: a randomised, controlled trial. Lancet Oncol. 2011;12(11):997-1003.

24. Kubben PL, Scholtes F, Schijns OE, et al. Intraoperative magnetic resonance imaging versus standard neuronavigation for the neurosurgical treatment of glioblastoma: a randomized controlled trial. Surg Neurol Int. 2014;5:70.

25. Jenkinson MD, Barone DG, Bryant A, et al. Intraoperative imaging technology to maximise extent of resection for glioma. Cochrane Database Syst Rev. 2018;1:CD012788.

26. Coburger J, Merkel A, Scherer M, et al. Low-grade glioma surgery in intraoperative magnetic resonance imaging: results of a multicenter retrospective assessment of the German Study Group for Intraoperative Magnetic Resonance Imaging. Neurosurgery. 2016;78(6):775-786.

27. Harris PA, Taylor R, Thielke R, et al. Research electronic data capture (REDCap) - a metadata-driven methodology and workflow process for providing translational research informatics support. J Biomed Inform. 2009;42(2):377-381.

28. Sylvester PT, Evans JA, Zipfel GJ, et al. Combined high-field intraoperative magnetic resonance imaging and endoscopy increase extent of resection and progression-free survival for pituitary adenomas. Pituitary. 2015;18(1):72-85.

29. Reponen E, Tuominen H, Korja M. Evidence for the use of preoperative risk assessment scores in elective cranial neurosurgery: a systematic review of the literature. Anesth Analg. 2014;119(2):420-432.

30. Young J, Badgery-Parker T, Dobbins T, et al. Comparison of ECOG/WHO performance status and ASA score as a measure of functional status. J Pain Symptom Manage. 2015; 49(2):258-264.

31. Kikinis R, Pieper SD, Vosburgh KG. 3D Slicer: a platform for subject-specific image analysis, visualization, and clinical support. In: Jolesz FA, ed. Intraoperative Imaging and Image-Guided Therapy. Springer New York; 2014:277-289.

32. Trifiletti DM, Alonso C, Grover S, et al. Prognostic implications of extent of resection in glioblastoma: analysis from a large database. World Neurosurg. 2017;103:330-340.

33. Kuhnt D, Becker A, Ganslandt O, et al. Correlation of the extent of tumor volume resection and patient survival in surgery of glioblastoma multiforme with high-field intraoperative MRI guidance. Neuro Oncol. 2011;13(12):1339-1348.

34. Coburger J, Segovia von Riehm J, Ganslandt O, et al. Is there an indication for intraoperative MRI in subtotal resection of glioblastoma? A multicenter retrospective comparative analysis. World Neurosurg. 2018;110:e389-e397.

35. Rahman M, Abbatematteo J, De Leo EK, et al. The effects of new or worsened postoperative neurological deficits on survival of patients with glioblastoma. J Neurosurg. 2017;127(1): $123-131$.

36. Stummer W, Pichlmeier U, Meinel T, et al. Fluorescenceguided surgery with 5-aminolevulinic acid for resection of malignant glioma: a randomised controlled multicentre phase III trial. Lancet Oncol. 2006;7(5):392-401.

37. Roder C, Bisdas S, Ebner FH, et al. Maximizing the extent of resection and survival benefit of patients in glioblastoma surgery: high-field iMRI versus conventional and 5-ALAassisted surgery. Eur J Surg Oncol. 2014;40(3):297-304.

38. Coburger J, Hagel V, Wirtz CR, König R. Surgery for glioblastoma: impact of the combined use of 5-aminolevulinic acid and intraoperative MRI on extent of resection and survival. PLoS One. 2015;10(6):e0131872.

39. Coburger J, Scheuerle A, Kapapa T, et al. Sensitivity and specificity of linear array intraoperative ultrasound in glioblastoma surgery: a comparative study with high field intraoperative MRI and conventional sector array ultrasound. Neurosurg Rev. 2015;38(3):499-509.

40. Eljamel MS, Mahboob SO. The effectiveness and costeffectiveness of intraoperative imaging in high-grade glioma resection; a comparative review of intraoperative ALA, fluorescein, ultrasound and MRI. Photodiagn Photodyn Ther. 2016;16:35-43.

41. Abraham P, Sarkar R, Brandel MG, et al. Cost-effectiveness of intraoperative MRI for treatment of high-grade gliomas. Radiology. 2019;291(3):689-697.

42. McGirt MJ, Speroff T, Dittus RS, et al. The National Neurosurgery Quality and Outcomes Database ( $\left.\mathrm{N}^{2} \mathrm{QOD}\right)$ : general overview and pilot-year project description. Neurosurg Focus. 2013;34(1):E6.

43. Henker C, Hiepel MC, Kriesen T, et al. Volumetric assessment of glioblastoma and its predictive value for survival. Acta Neurochir (Wien). 2019;161(8):1723-1732.

44. Zhang Z, Jiang $\mathrm{H}$, Chen $\mathrm{X}$, et al. Identifying the survival subtypes of glioblastoma by quantitative volumetric analysis of MRI. J Neurooncol. 2014;119(1):207-214.

45. Molinaro AM, Hervey-Jumper S, Morshed RA, et al. Association of maximal extent of resection of contrast-enhanced and non-contrast-enhanced tumor with survival within molecular subgroups of patients with newly diagnosed glioblastoma. JAMA Oncol. 2020;6(4):495-503. 


\section{Disclosures}

Dr. Chicoine received funding from the following sources: 1) IMRIS, Inc., for an unrestricted educational grant to support an iMRI database and outcomes analysis project, the IMRIS Multicenter intraoperative MRI Neurosurgery Database (I-MiND); 2) The Head for the Cure Foundation; and 3) Mrs. Carol Rossfeld and The Alex \& Alice Aboussie Family Charitable Foundation. REDCap database supported by Clinical and Translational Science Award (CTSA) Grant [UL1 TR000448] and Siteman Comprehensive Cancer Center and NCI Cancer Center Support Grant P30 CA091842. Dr. Dunn is a cofounder with equity in Immunovalent. Dr. Jensen is a consultant for Medtronic. Dr. Kim is a consultant for Monteris Medical and receives support of non-study-related clinical or research effort that he oversees from Monteris Medical, Stryker, and Collagen Matrix. Dr. Leuthardt has direct stock ownership in Neurolutions, Sora Imaging Solutions, Osteovantage, Immunovalent, Inner Cosmos, Face to Face Biometrics, and Caeli Vascular. He is a consultant for Monteris and Sante Ventures. Dr. Oswood is in the speakers' bureau for Philips Healthcare. The authors have no personal financial or institutional interest in any of the drugs, materials, or devices described in this article.

\section{Author Contributions}

Conception and design: Chicoine, A Shah, Sylvester, Dunn, Evans, Leuthardt, Dacey, Kim, Grubb, Zipfel, Oswood, Jensen, Sutherland, Cahill, Abram, Honeycutt, M Shah. Acquisition of data: Chicoine, A Shah, Sylvester, Yahanda, Dunn, Evans, Rich, Dowling, Leuthardt, Dacey, Kim, Grubb, Zipfel, Oswood, Jensen, Sutherland, Cahill, Abram, Honeycutt, M Shah. Analysis and interpretation of data: Chicoine, A Shah, Sylvester, Yahanda, Vellimana, Dunn, Dacey, Tao. Drafting the article: Chicoine, A Shah, Sylvester, Yahanda, Vellimana, Dunn. Critically revising the article: Chicoine, A Shah, Sylvester, Yahanda, Vellimana, Dunn, Rich, Dowling, Leuthardt, Dacey, Kim, Grubb, Zipfel, Oswood, Jensen, Sutherland, Cahill, Abram, Honeycutt, M Shah, Tao. Reviewed submitted version of manuscript: all authors. Approved the final version of the manuscript on behalf of all authors: Chicoine. Statistical analysis: A Shah, Sylvester, Tao. Administrative/technical/material support: Chicoine, A Shah, Sylvester, Yahanda, Vellimana, Evans. Study supervision: Chicoine, Dunn, Dacey, Grubb, Zipfel, Oswood, Jensen, Sutherland, Cahill, Abram, Honeycutt, M Shah.

\section{Supplemental Information Previous Presentations}

An earlier version of this work was presented as an oral presentation as the 2018 Congress of Neurological Surgeons (Houston, TX, October 6-10, 2018).

\section{Correspondence}

Michael R. Chicoine: Washington University School of Medicine, St. Louis, MO. chicoinem@wudosis.wustl.edu. 